\title{
RELAÇÃO DO ÍNDICE DE MASSA CORPÓREA E SOMATÓRIO DE DOBRAS CUTÂNEAS COM O NÍVEL DE ATIVIDADE FÍSICA DE ESCOLARES
}

\section{RELATION OF THE BODY MASS INDEX AND SUM OF CUTANEOUS FOLDS TO THE LEVEL OF PHYSICAL ACTIVITY OF STUDENTS}

\author{
Renato Canevari Dutra da Silva ${ }^{1}$ \\ Débora Bernardes Peixoto ${ }^{2}$ \\ Laís Zanutim Pereira ${ }^{3}$ \\ Mônica Maciel Guimarães ${ }^{4}$ \\ Marcelo Gomes Judice ${ }^{5}$ \\ Fernando Duarte Cabral ${ }^{6}$
}

Resumo: O objetivo do estudo foi verificar possíveis relações entre o índice de massa corpórea (IMC), o somatório de dobras cutâneas (SDC) e o nível de atividade física de estudantes com idade entre 15 e 17 anos. Foi avaliado o nível de atividade física por meio do Questionário Internacional de Atividade Física (IPAQ) de 1229 adolescentes escolares com média de idade de 15,9 anos (DP= + 0,81), além do IMC e o SDC (tricipital e subescapular). Por intermédio do IPAQ, foi encontrado um percentual de $77,7 \%$ de adolescentes ativos e 22,3\% de adolescentes insuficientemente ativos. Quanto à avaliação do IMC, a amostra obteve uma média de $21,77 \mathrm{~kg} / \mathrm{m} 2$, com desvio padrão de $+3,63$. A rede privada de ensino apresentou uma média maior, estatisticamente significativa $(p=0,011)$ em relação à rede pública de ensino, no entanto, ambas apresentam-se dentro dos parâmetros de normalidade, em relação à média de idade, conforme os percentis do CDC (2000). Quanto ao SDC, observou-se que a amostra obteve uma média de $31,83 \mathrm{~mm}$, com desvio padrão de $\pm 12,30$, com relação às redes de ensino, nota-se que não houve diferença estatisticamente significativa entre as médias, porém ambas apresentam-se elevadas, de acordo com a classificação de Lohman (1992). Não foi observada relação estatisticamente significativa entre o IMC dos adolescentes e nível de atividade física, no entanto, o SDC é maior nos adolescentes escolares com menor nível de atividade física.

Palavras-chave: Adiposidade corporal; adolescentes; composição corporal; exercício físico; medidas antropométricas.

Abstract: The aim of this study was to verify possible relations regarding the level of physical activity of students between 15 and 17 years old, the body mass index (BMI) and the sum of skinfold thickness (SST). The level of physical activity was evaluated by the International Questionnaire of Physical Activity (IPAQ) of 1229 students (15.9 years old, $S D=+0.81$ ), besides the index of body mass and the sum of cutaneous folds (tricipital and sub-scapular). By IPAQ, a percentage of $77.7 \%$ of active adolescents and $22.3 \%$ of insufficiently active adolescents were registered. Concerning BMI evaluation, the sample obtained an average of $21.77 \mathrm{~kg} / \mathrm{m} 2$ with a standard deviation of +3.63 . The private education students presented a higher average, statistically significant $(p=0.011)$ in relation to the public education students; however, both stay inside the normality parameters, in relation to the average of age, according to the percentiles of CDC (2000). Concerning SST, it was observed that the sample obtained an average of $31.83 \mathrm{~mm}$, with a standard deviation of \pm 12.30 ; in relation to both types of educational systems, no statistically significant difference was observed between the averages; however, both were elevated according to the Lohman classification

\footnotetext{
${ }^{1}$ Mestre em Ciências da Saúde/Universidade de Rio Verde - UniRV, Brasil. E-mail: renatocanevari@yahoo.com.br.

2 Graduanda em Medicina/Universidade de Rio Verde - UniRV, Brasil. E-mail: debora.bernardesp@hotmail.com.

${ }^{3}$ Graduanda em Medicina/Universidade de Rio Verde - UniRV, Brasil. E-mail: laiszanutim@gmail.com.

${ }^{4}$ Graduanda em Medicina/Universidade de Rio Verde - UniRV, Brasil. E-mail: mmacielguimaraes@gmail.com.

5 Professor Adjunto III/Universidade de Rio Verde - UniRV, Brasil. E-mail: mgjudice@gmail.com.

6 Fisioterapeuta na UTI e Diretor Administrativo - Hospital Municipal de Rio Verde/GO, Brasil. E-mail: fernandofisio2@hotmail.com.
} 
(1992). The level of physical activity was high in the adolescents evaluated (77.7\%) and no statistically significant difference was observed in the BMI of scholar adolescents concerning the level of physical activity; nevertheless, SST is higher in the school adolescents with a lower level of physical activity.

Keywords: Adolescents; anthropometric measurements; body adiposity; body composition; physical exercise.

\section{INTRODUÇÂO}

A obesidade pode ser definida, de forma simplificada, como uma doença caracterizada pelo acúmulo excessivo de gordura corporal, sendo consequência do balanço energético positivo o qual pode acarretar prejuízos à saúde dos indivíduos (FONTAINE et al., 2003; REIS, 2009). O excesso de gordura corporal pode ser acompanhado por uma variedade de disfunções crônico-degenerativas que elevam, consideravelmente, os índices de morbidade e mortalidade. (GUEDES et al., 2001; KIM et al., 2016).

Este estudo tem ainda como objetivo verificar a relação da obesidade com níveis de atividade física, já que a obesidade está intimamente relacionada ao estilo de vida do indivíduo. $O$ peso corporal aumenta quando a ingestão calórica ultrapassa o dispêndio de energia diário, ou seja, quando há ganho energético sem a sua utilização. Além disso, a obesidade representa, acima de tudo, um grave fator de risco para o desenvolvimento de doenças cardiovasculares e diabetes mellitus. $E$ a atividade física regular diminui tais riscos, aumentando a taxa de HDL-colesterol e diminuindo o LDLcolesterol, além de aumentar a ação da insulina.

A prevalência de obesidade entre crianças e adolescentes tem se tornado um grave problema de saúde e, atualmente, é estimada como sendo a quinta principal causa de mortalidade no mundo. (HAN et al., 2016). Na adolescência, o excesso de gordura corporal predispõe ao isolamento e afastamento das atividades sociais, como lazer e prática de atividade física, devido à discriminação decorrente do padrão de beleza imposto pela sociedade, mas também pela limitação de movimento, principalmente nas atividades esportivas. (MATSUDO et al., 2002). Tais limitações acarretam problemas respiratórios, diabetes mellitus, doenças cardiovasculares, dislipidemias, degenerações e instabilidades articulares, entre outros distúrbios. (DAMIANI; CARVALHO; OLIVEIRA, 2000; STYNE, 2001; POTHURAJU et al., 2013; KUMAR et al., 2012). Dessa forma, a avaliação da quantidade absoluta e percentual de gordura corporal em adolescentes torna-se um importante instrumento para um encaminhamento médico mais eficaz no diagnóstico de tais distúrbios.

Para a quantificação ou estimação da composição corporal, as medidas antropométricas, como o IMC, circunferência abdominal e circunferência de quadril têm sido amplamente utilizadas, devido à simplicidade, boa reprodutibilidade e baixo custo operacional. Ademais, o método antropométrico é recomendável para estudos e avaliações que envolvam um grande número de indivíduos, por ser um método não invasivo. (GIUGLIANO; MELO, 2004; LEÃO et al., 2003). 
O IMC por idade tem-se mostrado um método adequado para o diagnóstico de sobrepeso e obesidade em grupos populacionais de adolescentes. Tal método apresenta boa concordância com a adiposidade corporal, que pode ser verificada pelo cálculo das dobras cutâneas e quantificar a gordura interna, a qual está associada aos fatores de risco para doenças cardiovasculares. (HIMES; DIETZ, 1994).

As curvas de crescimento baseadas no IMC, foram revisadas pelo Centers for Disease Control and Prevention (CDC) (CDC, 2000) e incluíram valores de IMC, específicos para idade e sexo, para crianças, adolescentes e jovens adultos com a utilização de percentis que variam de abaixo de três, para indivíduos com baixo IMC, de três a oitenta e quatro IMC, normal; de oitenta e cinco a noventa e quatro, sobrepeso; e igual ou maior que noventa e cinco, para obesidade.

Outro método antropométrico utilizado na avaliação da composição corporal é a medida de dobras cutâneas. A mensuração de dobras cutâneas é um método que estima a adiposidade existente no tecido subcutâneo. Já, o IMC não faz distinção sobre a distribuição da gordura corporal entre os diferentes compartimentos; além disso, o IMC não avalia a constituição do corpo, sendo essa gordura, massa magra ou osso. (MONTEIRO et al., 2000).

A avaliação do nível de atividade física é relevante para os estudos em epidemiologia e ciências aplicadas ao exercício, sobretudo quando o objetivo é a prevenção das doenças crônicas não transmissíveis. Mas são escassos os instrumentos para a avaliação da atividade física na população brasileira, principalmente para crianças e adolescentes. (FLORINDO et al., 2006).

Nessa idade, a atividade física contribui para redução da obesidade, e melhora os perfis lipídico e metabólico. É comprovado que crianças ativas tornam-se adultos mais saudáveis. Assim, promover atividade física na infância e adolescência estabelece uma base sólida para a redução do sedentarismo em adultos e reduzir o aparecimento de doenças, melhorando a qualidade de vida. (MATSUDO et al., 2002; SILVA et al., 2013; VACCARO, 1989). A atividade física melhora a aptidão física, performance, otimiza o crescimento e estimula a participação em programas de atividade física e sociais. (MATSUDO et al., 2002).

Uma das alternativas para estimar o nível da prática de atividade física em grupos populacionais é a utilização do Questionário Internacional de Atividade Física (IPAQ), que foi proposto pelo Grupo Internacional para Consenso em Medidas da Atividade Física. O IPAQ foi desenvolvido com a finalidade de estimar o nível de prática habitual de atividade física de populações de diferentes países e contextos socioculturais. (MATSUDO et al., 2001).

Estudos que relacionam o IMC e somatório das dobras cutâneas (SDC) com o nível de atividade física de crianças e adolescentes são importantes, pois a obesidade é uma doença grave, que vem sendo considerada como um grande problema de saúde 
pública. Assim como a obesidade, a inatividade física é um importante fator de risco para doenças crônicas não transmissíveis e, juntas, acarretam maiores complicações ao indivíduo. (BRITO et al., 2012).

O objetivo do estudo foi verificar possíveis relações entre o índice de massa corpórea, o somatório de dobras cutâneas e o nível de atividade física de escolares. A hipótese é que crianças e adolescentes com níveis de atividade física baixos apresentam valores de IMC e SDC alterados, indicando maiores riscos para enfermidades relacionadas com níveis de gordura corporal.

\section{METODOLOGIA}

Trata-se de um estudo descritivo e transversal. A amostra foi composta por 1229 adolescentes de ambos os sexos, com média de idade de 15,9 anos $(+0,81)$, dos quais 466 alunos $(37,9 \%)$ pertenciam à rede privada e $763(62,1 \%)$ à rede pública de ensino, distribuídos em 11 escolas do município de Rio Verde - GO, no segundo semestre de 2012, nos meses de novembro e dezembro.

O presente estudo foi aprovado pelo Comitê de Ética em Pesquisa com Seres Humanos da UNITRI - Centro Universitário do Triângulo, com o número do parecer 598771 (sob o título "Obesidade, perfil lipídico e sua relação com o nível de atividades física de adolescentes escolares").

As escolas estudadas continham, no total, 3063 adolescentes cursando o ensino médio, no período matutino, sendo que desses, 2275 adolescentes pertenciam às escolas da rede pública, distribuídos num total de 5 escolas, e 788 adolescentes pertenciam a 6 escolas privadas.

Todos os adolescentes foram informados a respeito dos objetivos e procedimentos da pesquisa por intermédio do Termo de Assentimento, que foi assinado por aqueles que aceitaram a participar da pesquisa, posteriormente foi encaminhado o Termo de Consentimento Livre e Esclarecido (TCLE) aos seus pais. Ademais, foram submetidos aos critérios de inclusão, os quais são: serem regularmente matriculados no ensino médio, no período matutino do município de Rio Verde - GO e terem idade entre 15 e 17 anos. Foram excluídos os alunos que apresentavam idade menor que 14 ou maior que 18 anos, que não estiveram presentes na sala de aula, no dia da realização da pesquisa e os quais não apresentaram o TCLE ou que se recusaram a participar da pesquisa.

Posteriormente, foram avaliados a composição corporal por meio do IMC; do método somatório de dobras cutâneas Tricipital (T) e a Subescapular (SE); e do nível de atividade física por meio da versão curta do IPAQ. O questionário foi respondido no horário e ambiente escolar, sem a presença do professor e, posteriormente, os adolescentes foram avaliados com relação às possíveis doenças pré-existentes que 
pudessem interferir na avaliação do nível de atividade física.

Para o cálculo do IMC, foi utilizada a equação massa corporal sobre o quadrado da estatura $\{\mathrm{IMC}=$ massa corporal $(\mathrm{Kg}) /$ Estatura2 $(\mathrm{m})\}$. A massa corporal e a estatura foram medidas conforme descrito por Pitanga (2005). A medida do peso corporal $(\mathrm{Kg})$ foi obtida uma única vez, com os alunos descalços e vestindo short e camiseta com o mínimo de roupa possível, em balança antropométrica (Indústria Fillizola S.A - Brasil), com capacidade de 0-150 kg e precisão de $100 \mathrm{~g}$, devidamente aferida (INMETRO) (FRANÇA; VÍVOLO, 1987). Para coleta da estatura, foi utilizado o estadiômetro vertical da própria balança, com capacidade de $2 \mathrm{~m}$ e precisão de 0,1 centímetros $(\mathrm{cm})$, com os escolares descalços, em posição ereta, com calcanhares unidos e pés com as pontas afastadas, braços pendentes e com as mãos espalmadas sobre as coxas, cabeça ajustada ao Plano de Frankurt em inspiração profunda. (GORDON; CHUMLEA; ROCHE, 1988). Posteriormente, o IMC foi classificado de acordo com a idade.

O critério de referência para definir as variáveis relacionadas ao perfil nutricional de crianças de adolescentes foi a classificação do CDC (Centers for Disease and Control and Prevention), (CDC, 2000) recomendada pela Associação Brasileira de Nutrologia (ABRAM), que é baseada no IMC abaixo de 18,5, para baixo peso; de 18,5 a 24,9, peso normal; de 25,0 a 29,9, sobrepeso; e maior ou igual a 30,0 , obeso.

A estimativa de gordura corporal foi obtida a partir da medida da espessura das dobras cutâneas tricipital (T) e Subescapular (SE). As dobras cutâneas foram medidas por meio do adipômetro Sanny, com precisão de 0,5 milímetros $(\mathrm{mm})$. Os escolares estavam em posição ortostática, olhando para frente e com os membros superiores relaxados. A dobra $\mathrm{T}$ foi medida no ponto médio entre a borda superolateral do acrômio e o olecrano e a SE foi obtida obliquamente ao eixo longitudinal, seguindo a orientação dos arcos costais, localizada $2 \mathrm{~cm}$ abaixo do ângulo inferior da escápula. (FERNANDES FILHO, 2003).

O índice de adiposidade corporal foi obtido de acordo com a classificação proposta por Lohman, que é dado pelo somatório das dobras T e SE e comparados com os valores de referência, de acordo com a tabela 1 (LAZZOLI et al., 1998).

Para a análise estatística, verificaram-se a normalidade dos dados e a prevalência da obesidade. Foi utilizada a amplitude (mínimo e máximo) e o cálculo percentual das variáveis dependentes IMC. Em adição, a estatística inferencial foi utilizada por meio do Teste T de Student e Teste binomial, para verificar possíveis diferenças e proporções no nível de atividade física, IMC e o SDC. Os dados foram apresentados em média $( \pm$ Desvio Padrão; DP) e significância de $p \leq 0,05$.

No estudo de validação do Questionário Internacional de Atividade Física (IPAQ), nas versões curta e longa, afirmam não haver diferença da forma de aplicação entre a entrevista feita por telefone e questionário auto-aplicável e acrescentaram que esse tipo de instrumento é válido e reprodutível nas duas versões. Em relação à idade, a pesquisa 
foi realizada em indivíduos acima de 12 anos, não apresentando nenhum tipo de contratempo descrito na pesquisa, sendo possível estabelecer o nível de atividade física nessa idade, por meio de questionário. (MATSUDO et al., 2001).

Tabela 1 - Classificação do índice de adiposidade corporal proposta por Lohman para crianças e adolescentes.

\begin{tabular}{lcccc}
\hline & \multicolumn{2}{c}{ MENINOS } & \multicolumn{2}{c}{ MENINAS } \\
& DC(mm) T+SE & $\% \mathrm{G}$ & $\mathrm{DC}(\mathrm{mm}) \mathrm{T}+\mathrm{SE}$ & $\% \mathrm{G}$ \\
\hline Muito Baixo & 5 & 8 & $5-10$ & $7-11$ \\
Baixo & 10 & 10 & 15 & 14 \\
Nível Ótimo & $15-25$ & $13-20$ & $20-30$ & $18-25$ \\
Moderadamente Alto & $25-30$ & $20-24$ & $30-35$ & 29 \\
Alto & $35-40$ & $28-31$ & $40-45$ & $32-38$ \\
Muito Alto & $40-55$ & $31-42$ & $50-55$ & $39-43$
\end{tabular}

DC= Dobras cutâneas: $T=$ tricipital; $S E=$ subescapular; \%G=porcentagem de gordura.

\section{RESULTADOS}

Ao fim da análise estatística, observou-se que 47,2\% dos avaliados eram do sexo masculino e de $52,8 \%$ do sexo feminino. A tabela 2 representa os resultados estatísticos do índice de massa corpórea, bem como o somatório de dobras cutâneas dos adolescentes avaliados.

Com relação à avaliação do IMC dos adolescentes $(n=1229)$, observa-se que a amostra obteve uma média de $21,77 \mathrm{~kg} / \mathrm{m} 2$, com desvio padrão de $+3,63$, sendo o menor IMC de $14,58 \mathrm{~kg} / \mathrm{m} 2$ e o maior de $43,59 \mathrm{~kg} / \mathrm{m} 2$.

Quanto ao SDC (tabela 2), observa-se que a amostra obteve uma média de 31,83 $\mathrm{mm}$, com desvio padrão de $\pm 12,30$, sendo o somatório mínimo de $10,0 \mathrm{~mm}$ e o máximo de $78,0 \mathrm{~mm}$.

Em relação à classificação geral do IPAQ para toda a amostra $(n=1229)$, apresentou o percentual de 2,30\% de adolescentes sedentários (S); 9,60\%, insuficientemente ativo B (IB); 10,40\%, como insuficientemente ativo A (IA); e com maiores percentuais obtidos para adolescentes ativos (A), com $51,30 \%$; e muito ativos (MA), $26,40 \%$.

Para melhor análise dos dados, as cinco classificações do IPAQ foram compactadas em apenas duas: sedentários e insuficientemente ativos $A$ e $B$, foram classificados de insuficientemente ativos; para ativos e muito ativos, foram denominados de ativos. Assim, esta integração representou $77,7 \%$ de adolescentes ativos, e 22,3\% insuficientemente ativos. 
Tabela 2 - Descrição dos valores do IMC e SDC dos adolescentes de forma Geral em relação às redes de ensino.

\begin{tabular}{llllllllll}
\hline VARIAVEIS & & N & Mínimo & Máximo & Média & $\begin{array}{l}\text { Desvio } \\
\text { Padrão }\end{array}$ & t & p \\
& & & & & & & \\
\hline IMC & Geral & 1229 & 14,58 & 43,59 & 21,7722 & 3,63571 & - & - \\
& RPV & 466 & 14,58 & 39,88 & 22,1078 & 3,65588 & 2,534 & $0,011^{*}$ \\
& RPB & 763 & 14,70 & 43,59 & 21,5673 & 3,61041 & & \\
SDC & Geral & 1229 & 10,0 & 78,0 & 31,834 & 12,3096 & - & - \\
& RPV & 466 & 10,0 & 75,1 & 31,548 & 10,9329 & $-0,637$ & 0,524 \\
& RPB & 763 & 10,0 & 78,0 & 32,009 & 13,0832 & & \\
\hline
\end{tabular}

RPV = Rede Privada; RPB = Rede Pública; * diferença estatisticamente significativa.

Figura 1. Percentuais de adolescentes insuficientemente ativos e ativos.

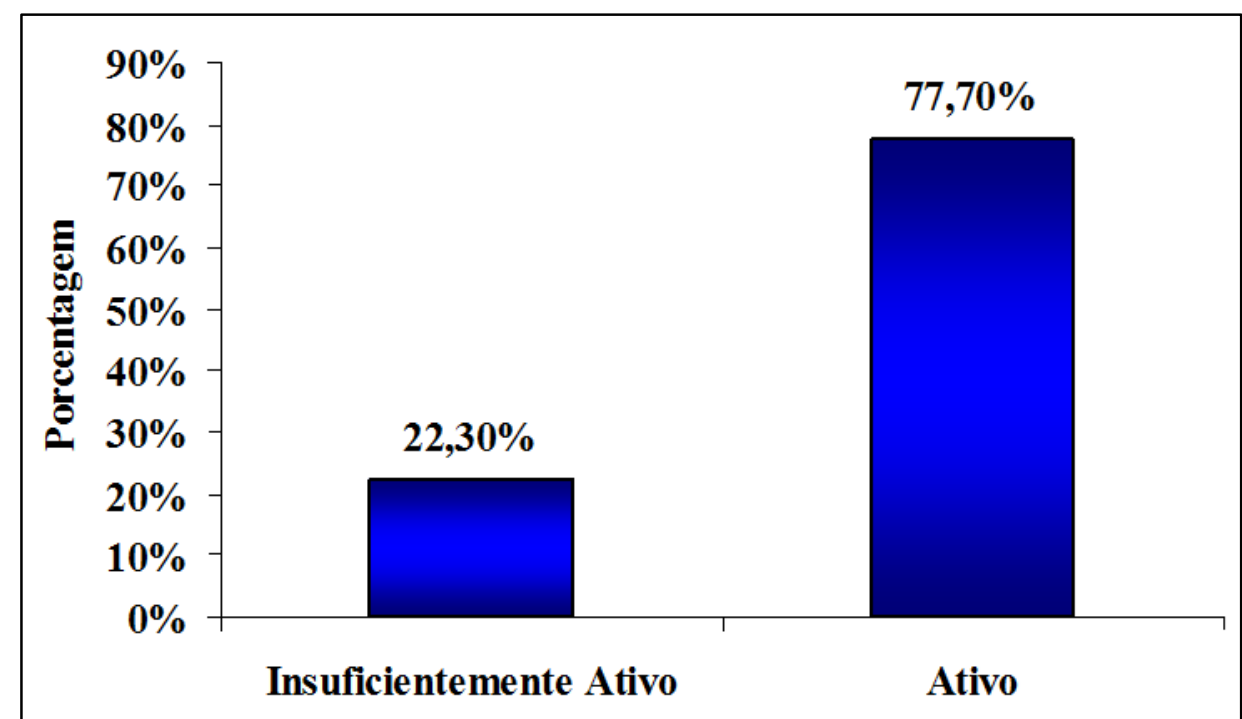

Em relação ao IMC, de acordo com a classificação do IPAQ (Tabela 3), não houve diferença estatisticamente significativa $(p=0,0901)$ entre o nível de atividade física e o IMC; no entanto, a média do IMC foi maior nos adolescentes ativos, já os adolescentes insuficientemente ativos apresentaram o SDC maior que os adolescentes ativos ( $p=$ 0,0001). 
Tabela 3 - Estatísticas inferenciais do IMC e SDC de acordo com o nível de atividade física em relação às redes de ensino.

\begin{tabular}{|c|c|c|c|c|c|c|c|c|}
\hline & & IPAQ & $\mathbf{N}$ & Mínimo & Máximo & Média & $\begin{array}{l}\text { Desvio } \\
\text { Padrão }\end{array}$ & $\mathbf{p}$ \\
\hline & $\begin{array}{l}\text { IMC } \\
(\mathrm{kg} / \mathrm{m})\end{array}$ & $\begin{array}{l}\text { Insuficiente } \\
\text { Ativo } \\
\text { Ativo }\end{array}$ & $\begin{array}{r}274 \\
955\end{array}$ & $\begin{array}{r}14,58 \\
14.70\end{array}$ & $\begin{array}{r}38,38 \\
43,59\end{array}$ & $\begin{array}{r}21,80 \\
21,77\end{array}$ & $\begin{array}{l}3,82 \\
3,58\end{array}$ & 0,901 \\
\hline Geral & $\begin{array}{l}\text { SDC } \\
(\mathrm{mm})\end{array}$ & $\begin{array}{l}\text { Insuficiente } \\
\text { Ativo } \\
\text { Ativo }\end{array}$ & $\begin{array}{r}274 \\
955\end{array}$ & $\begin{array}{l}13,0 \\
10,0\end{array}$ & $\begin{array}{l}78,0 \\
78,0\end{array}$ & $\begin{array}{r}34,78 \\
30,99\end{array}$ & $\begin{array}{r}13,33 \\
11,87\end{array}$ & $0,0001^{*}$ \\
\hline $\begin{array}{l}\text { Rede } \\
\text { Pública }\end{array}$ & $\begin{array}{l}\mathrm{IMC} \\
\left(\mathrm{kg} / \mathrm{m}^{2}\right)\end{array}$ & $\begin{array}{l}\text { Insuficiente } \\
\text { Ativo } \\
\text { Ativo }\end{array}$ & $\begin{array}{l}139 \\
624\end{array}$ & $\begin{array}{r}15,79 \\
14,70\end{array}$ & $\begin{array}{r}35,70 \\
43,59\end{array}$ & $\begin{array}{l}22,04 \\
21,46\end{array}$ & $\begin{array}{l}3,85 \\
3,55\end{array}$ & 0,089 \\
\hline Pública & $\begin{array}{l}\text { SDC } \\
(\mathrm{mm})\end{array}$ & $\begin{array}{l}\text { Insuficiente } \\
\text { Ativo } \\
\text { Ativo }\end{array}$ & $\begin{array}{r}139 \\
624\end{array}$ & $\begin{array}{l}13,0 \\
10,0\end{array}$ & $\begin{array}{l}78,0 \\
78,0\end{array}$ & $\begin{array}{l}35,85 \\
31,15\end{array}$ & $\begin{array}{l}15,02 \\
12,46\end{array}$ & $0,0001^{*}$ \\
\hline $\begin{array}{l}\text { Rede } \\
\text { Privada }\end{array}$ & $\begin{array}{l}\mathrm{IMC} \\
\left(\mathrm{kg} / \mathrm{m}^{2}\right)\end{array}$ & $\begin{array}{l}\text { Insuficiente } \\
\text { Ativo } \\
\text { Ativo }\end{array}$ & 135 & 14,58 & 38,38 & 21,55 & $\begin{array}{l}3,78 \\
3,58\end{array}$ & $0,035^{*}$ \\
\hline Privada & $\begin{array}{l}\text { SDC } \\
(\mathrm{mm})\end{array}$ & $\begin{array}{l}\text { Insuficiente } \\
\text { Ativo } \\
\text { Ativo }\end{array}$ & 135 & 14,0 & 75,1 & 33,69 & $\begin{array}{r}11,29 \\
10,68\end{array}$ & $0,007^{*}$ \\
\hline
\end{tabular}

* diferença estatisticamente significativa.

\section{DISCUSSÃO}

No presente estudo, observou-se que a rede privada de ensino apresenta uma média maior de IMC e SDC, estatisticamente significativa $(p=0,011)$ em relação à rede pública; no entanto, ambas apresentam-se dentro dos parâmetros de normalidade, em relação à média de idade, conforme os percentis do Centers for Disease Control and Prevention CDC (CDC, 2000).

Os resultados obtidos em relação ao SDC, permitem-nos dizer que é relevante o estímulo da prática de atividade física, de acordo com os critérios do CDC. (GUEDES et al., 2001).

O Instituto Nacional do Câncer (INCA), (INSTITUTO..., 2004), realizou um inquérito domiciliar em 15 capitais brasileiras, verificando maior percentual de indivíduos insuficientemente ativos, no município de João Pessoa (54,5\%), e menor, em Belém $(27,4 \%)$. No estado de São Paulo, $46,5 \%$ dos indivíduos eram sedentários e em PelotasRS detectaram que $41,1 \%$ dos indivíduos eram sedentários. (HALLAL et al., 2003). Esses dados não se assemelham ao presente estudo.

A prevalência de adolescentes ativos deste estudo é superior que as encontradas 
na maioria dos estudos, contudo foi similar à prevalência de adolescentes ativos encontrados em Natal-RN (74,1\%), (INCA, 2004), sendo possível que o fator regional, sobretudo o climático, possa ter interferido satisfatoriamente sobre os percentuais de adolescentes ativos. Em investigação sobre os fatores que interferem na prática de exercícios, discutem que no verão os indivíduos tendem a praticar mais atividade física. (MCGUIRE et al., 2002). Simultaneamente, em Rio Verde - GO, apresentam elevadas temperaturas, com média de 20 a $35^{\circ} \mathrm{C}$.

Os adolescentes insuficientemente ativos apresentaram o SDC significativamente ( $p=0,0000$ ) maior que os ativos, o que implica que os menos ativos, possuem maior quantidade de tecido adiposo subcutâneo e a atividade física é fator determinante com relação ao peso corporal, contribuindo para a redução de gordura, aumento de massa muscular e densidade óssea.

Outro estudo corrobora com este, pois detectou uma prevalência de $39 \%$ de escolares femininos de Florianópolis-SC, que estavam acima dos níveis ótimos de adiposidade e o grupo de indivíduos com menor adiposidade corporal eram mais ativos que o grupo acima dos níveis ótimos de gordura corporal. (LOBO; LOPES, 2001).

O comportamento sedentário entre crianças e adolescentes pode ser um fator agravante para o acúmulo de gordura corporal, contribui para a obesidade, enquanto que um aumento nos níveis diários de atividade física pode reduzir a adiposidade corporal e seus efeitos adversos. (PINHO; PETROSKI, 1999).

A obesidade está mais associada à inatividade física do que aos excessos alimentares (POLOCK; WILMORE, 1993), além do que, jovens obesos são fisicamente menos ativos que seus pares classificados em níveis ótimos de adiposidade corporal. Em estudo longitudinal, foi constatado que os adolescentes com baixos níveis de atividade física ganharam mais gordura corporal, comparados aos que tinham um estilo de vida mais ativo. (NEUTZLING et al., 2000).

Diante desses dados, parece que intervenções estratégicas são necessárias para promover um estilo de vida mais ativo entre adolescentes escolares e, assim, reverter os riscos para a saúde, evitar um comportamento sedentário e manter o nível de atividade física dos adolescentes ativos, pois o percentual de adolescentes insuficientemente ativos neste estudo foi de $22,3 \%$.

$\mathrm{Na}$ rede pública de ensino, foi observado que não houve diferença estatisticamente significativa, na relação entre o IMC e o SDC, entre os adolescentes insuficientemente ativos e nos ativos com relação ao IMC, mas ao SDC foi observada diferença estatística significante maior nos insuficientemente ativos $(p=0,0001)$.

Na rede privada de ensino, adolescente com maior nível de atividade física o IMC foi maior ( $p=0,035$ ), já o SDC foi maior nos adolescentes com menor nível de atividade física $(p=0,007)$. Esses dados são explicados pela maior acessibilidade dos alunos da rede privada de ensino a clubes esportivos e academias de ginástica, o que permite 
maior participação em atividades que demandam no aumento da massa muscular. (GUEDES et al., 2001).

Esses resultados ocorrem, pois o IMC não faz separação dos diferentes tecidos, sendo ele a soma das variáveis corporais, como tecido ósseo, muscular, adiposo, enquanto as dobras cutâneas avaliam, realmente, a adiposidade existente no tecido subcutâneo (FLORINDO et al., 2006). Logo, o aumento do IMC nos adolescentes ativos do presente estudo pode estar relacionado ao ganho de massa muscular e não de tecido adiposo, uma vez que o SDC, nesses adolescentes, foi menor.

Ainda que os resultados aqui apresentados possam oferecer novos conhecimentos para a área de estudo, chama-se a atenção para as seguintes limitações: (1) delineamento transversal, não possibilitando a relação de causalidade entre as variáveis (IMC, SBC, nível de atividade física de escolares); (2) disposição temporal quanto às modificações nos estágios de mudanças de comportamento que não puderam ser acompanhadas. Apesar das limitações inerentes ao estudo transversal, os dados coletados foram extraídos de uma amostra representativa de estudantes.

São necessários novos estudos sobre os possíveis fatores que possam modificar e influenciar o dispêndio energético e sua relação com a adiposidade para explicar o possível desequilíbrio entre as necessidades energéticas com o movimento, sua necessidade e os fatores que influenciam a diminuição do tecido adiposo.

\section{CONCLUSÃO}

Observou-se alto nível de atividade física dos adolescentes avaliados e quanto maior o nível de atividade física desses adolescentes menor a concentração de tecido adiposo subcutâneo, e menor a prevalência de obesidade.

Não foi observada diferença estatisticamente significativa no IMC dos adolescentes quanto ao nível de atividade física, no entanto, quanto ao SDC, este é maior nos adolescentes com menor nível de atividade física.

O IMC é maior nos alunos da rede privada de ensino considerados suficientemente ativos. O SDC foi elevado em ambas as redes de ensino, não verificando diferenças entre elas.

\section{REFERÊNCIAS}

BRASIL. Instituto Nacional do Câncer (INCA). Inquérito domiciliar sobre comportamento de risco e morbidade referida de doenças e agravos não transmissíveis. 2003. Disponível em:

<http://bvsms.saude.gov.br/bvs/publicacoes/inca/inquerito22_06_parte1.pdf>. Acesso em: 14 jun. 2016.

BRITO, A. K. A. et al. Nível de atividade física e correlação com o índice de massa 
corporal e percentual de gordura em adolescentes escolares da cidade de Teresina-PI. Revista Brasileira de Atividade Física e Saúde, v. 17, n. 3, p. 212-216, 2012.

CENTERS FOR DISEASE CONTROL AND PREVENTION et al. Promoting physical activity: a best buy in public health. A Report from the CDC. Atlanta: CDC, 2000.

DAMIANI, D.; CARVALHO, D. P.; OLIVEIRA, R. G. Obesidade na infância: um grande desafio. Pediatria Moderna, v. 36, n. 8, 2000. Disponível em: <http://www.sbp.com.br/img/documentos/doc_obesidade_inf\%E2ncia.pdf $>$. Acesso em: 13 jun. 2016.

FERNANDES FILHO, J. A prática da avaliação Física: Teses, medidas e Avaliação Física em Escolares Atletas e Academias de Ginástica. 2. ed. Rio de Janeiro: Shape, 2003.

FLORINDO, A. A. et al. Desenvolvimento e validação de um questionário de avaliação da atividade física para adolescentes. Revista Saúde Pública, v. 40, n. 5, p. 802-809, 2006.

FONTAINE, K. R. et al. Years of life lost due to obesity. Journal of the American Medical Association, v. 28, n. 9, p. 187-193, 2003.

FRANÇA, N. M.; VÍVOLO, M. A. Medidas antropométricas. In: MATSUDO, V. K. R. (Ed.) Testes em Ciências do Esporte. 6. ed. São Caetano do Sul: CELAFISCS, 1987. p. 19-31.

GIUGLIANO R.; MELO A. L. P. Diagnóstico de sobrepeso e obesidade em escolares: utilização do índice de massa corporal segundo padrão internacional. Jornal de Pediatria, v. 80, n. 2, p. 129-134, 2004.

GORDON, C. C.; CHUMLEA, W. C.; ROCHE, A. F. Stature, recumbent length, and weight. In: LOHMAN, T. G.; ROCHE, A. F.; MARTORELL, R. Anthropometric Standardization Reference Manual. Champaign: Human Kinetics Books, 1988. p. 38.

GUEDES, D. P. et al. Níveis de prática de atividade física habitual em adolescentes. Revista Brasileira de Medicina do Esporte, v. 7, n. 6, p. 187-199, 2001.

HALLAL, P. C. et al. Physical inactivity: prevalence and associated variables in Brazilian adults. Medicine \& Science in Sports \& Exercise, v. 35, n. 11, p. 18941900, 2003.

HAN, K. et al. Trends in obesity at the national and local level among South Korean adolescentes. Geospatial Health, v. 11, n. 2, p. 130-136, 2016.

HIMES, J. H.; DIETZ, W. H. Guidelines for overweight in adolescent preventive services: recommendations from an expert committee. American Journal of Clinical Nutrition, v. 59, n.2, p. 307-316, 1994.

INSTITUTO NACIONAL DO CÂNCER - (INCA). Inquérito domiciliar sobre comportamento de risco e morbidade referida de doenças e agravos não transmissíveis: Brasil, 15 capitais e Distrito Federal, 2002-2003. 2004. Disponível em: <http://bvsms.saude.gov.br/bvs/publicacoes/inca/inquerito22_06_parte1.pdf>. Acesso em: 14 jun. 2016.

$\mathrm{KIM}, \mathrm{H}$. et al. Effects of feeding a diet containg Gymnema sylvestre extract: Attenuating 
progression of obesity in C57BL/6J mice. Asian Pacific Journal of Tropical

Medicine, Seaul, v. 9, n. 5, p. 437-444, 2016.

KUMAR, V. et al. Evaluation of antiobesity and cardioprotective effect of Gymnema sylvestre extract in murine model. Indian Journal of Pharmacology, v. 44, n. 5, p. 607-613, 2012.

LAZZOLI, J. K. et al. Atividade física e saúde na infância e adolescência. Revista Brasileira de Medicina do Esporte, v. 4, n. 4, p. 107-109, 1998.

LEÃO, S. C. S. et al. Prevalência de obesidade em escolares de Salvador, Bahia. Arquivos Brasileiros de Endocrinologia e Metabologia, v. 47, n. 2, p. 151-157, 2003.

LOBO, A. S.; LOPES, A. S. Adiposidade corpórea e sua relação com o gasto energético e a composição da dieta de escolares do sexo feminino. Revista Brasileira Atividade física e saúde, v. 6, n. 2, p. 69-78, 2001.

LOHMAN, T. G. Advances in body composition assessment. Human Kinetics Publishers. 1992.

MATSUDO, S. et al. Questionário Internacional de Atividade Física (IPAQ): estudo de validade e reprodutibilidade no Brasil. Revista Atividade Física \& Saúde, v. 6, n. 2, p. 5-18, 2001.

MATSUDO S. M. et al. Nível de atividade física da população do Estado de São Paulo: análise de acordo com o gênero, idade, nível socioeconômico, distribuição geográfica e de conhecimento. Revista Brasileira de Ciência e Movimento, v. 10, n. 4, p. 41-50, 2002.

MCGUIRE, M. T. et al. Parental correlates of physical activity in a racially/ethnically diverse adolescent sample. Journal of Adolescent Health, v. 30, n. 4, p. 253-261, 2002.

MONTEIRO, P. O. A. et al. Diagnóstico de sobrepeso em adolescentes: estudo do desempenho de diferentes critérios para o Índice de Massa Corporal. Revista de Saúde Pública, v. 34, n. 5, p. 506-513, 2000.

NEUTZLING, M. B. et al. Overweight and obesity in Brazilian adolescents. International Journal of Obesity, v. 24, p. 1-7, 2000.

PINHO, R. A.; PETROSKI, E. L. Adiposidade corporal e nível de atividade física em adolescentes. Revista Brasileira de Cineantropometria e Desempenho Humano, $v$. 1, p. 60-68, 1999.

PITANGA, Francisco José Gondim. Testes, medidas e avaliação em educação física e esportes. Phorte, 2008.

POLLOCK, Michael L.; WILMORE, Jack H. Exercícios na saúde e na doença. Rio de Janeiro: Medsi, p. 231-605, 1993.

POTHURAJU, R. et al. A systematic review of Gymnema sylvestre in obesity and diabetes management. Journal of the Science of Food and Agriculture, v. 94, n. 5, p. 834-840, 2014. 
REIS, C. P. Obesidade e atividade física. EFDeportes. com, Revista Digital-Buenos Aires, n.130, 2009. Disponível em: <http://www.efdeportes.com/efd130/obesidade-eatividade-fisica.htm $\geq$. Acesso em:

SILVA, S. L. et al. Influência de fatores antropométricos e atividade física na pressão arterial de adolescentes de Taguatinga, Distrito Federal, Brasil. Motricidade, v. 9, n. 1, p. 13-22, 2013.

STYNE, D.M. Childhood and adolescent obesity: prevalence and significance.

Pediatric Clinics of North América, v. 48, n.4, p. 823-854, 2001. 